\title{
Atelectasia pulmonar recurrente secundaria a una metástasis endobronquial de un carcinoma de colon. Caso clínico
}

\author{
Cristian G odoy $\mathbf{T}^{1,3}$, Jorge Vega $\mathbf{S}^{1,3}$, Liliana Rivera $\mathbf{~}^{2}$. \\ Recurrent left lung atelectasis caused \\ by an endobronchial metastasis \\ of a colon cancer. Report of one case
}

Endobronchial location of metastases is uncommon. We report a 83 year-old woman with a history of a left hemicolectomy due to tubular colon adenocarcinoma, three years ago. She consulted in the emergency room for progressive dyspnea, cough an mucous sputum. There was abolition of breath sounds and dullness in the left hemithorax. Chest X ray examination showed a complete opacity of the left lung. She was treated as a pneumonia and her left lung expanded again. Three weeks later, left lung atelectasis relapsed. A bronchial biopsy showed a moderately differentiated adenocarcinoma, compatible with colon adenocarcinoma. Immunohistochemistry confirmed the colonic origin of the tumor. The patient rejected radiotherapy and is alive after 11 months of follow up (Rev Méd Chile 2008; 136: 217-20).

(Key words: Adenocarcinoma; Bronchial neoplasms; Colonic neoplasms)

Recibido el 22 de diciembre, 2006. Aceptado el 7 de junio, 2007.

${ }^{1}$ Servicio de Medicina Interna. ${ }^{2}$ Departamento de Anatomía Patológica, Hospital Naval Almirante Nef Viña del Mar. ${ }^{3}$ Departamento de Medicina. Escuela de Medicina. Universidad de Valparaíso.

$\mathrm{E}^{1}$ parénquima pulmonar es un sitio frecuente de metástasis de tumores malignos, pero la localización endobronquial (EB) de las metástasis es rara. En autopsias realizadas en sujetos con metástasis pulmonar, la localización EB se ha encontrado sólo en $2 \%$ de ellos $^{1}$. Los orígenes más frecuentes del tumor primario han sido: mama,

Correspondencia a: Dr. Cristián Godoy T. Eusebio Lillo 550, Depto. 802, Torre Valparaíso, Valparaíso. Fono: (5632) 2497865. E mail: cristiangt@gmail.com riñón, colon, recto, cuello uterino, tiroides, testículo y próstata. De éstos, los de colon y recto han sido responsables de las metástasis EB entre 4\% y $14 \% 2,3$. Una característica propia de esta ubicación es su tendencia a producir obstrucción de la vía aérea, tal como ocurre con el carcinoma broncogénico. Sólo la biopsia del tejido EB permite diferenciarlos.

Comunicamos el caso de una paciente de edad avanzada que debutó con una falla respiratoria secundaria a una atelectasia recurrente del pulmón izquierdo, originada en una metástasis de un carcinoma de colon. 


\section{Caso CLÍNICO}

Mujer de 83 años, no fumadora, hipertensa, con el antecedente de haber sido sometida a una hemicolectomía derecha por un adenocarcinoma tubular del colon tres años antes. No recibió radioterapia ni quimioterapia postoperatoria. Dos años después fue reparada una hernia incisional. En septiembre de 2005 ingresó a otro hospital por fiebre y disnea de reposo. Al examen físico destacó la ausencia de murmullo pulmonar a izquierda y los exámenes revelaron hipoxemia, hiperventilación, anemia (hematocrito 33\%), leucocitosis $\left(23.000 \mathrm{~mm}^{3}\right)$ y VHS elevada $(89 \mathrm{~mm} / \mathrm{h})$. Una radiografía de tórax mostró una atelectasia completa del pulmón izquierdo. Se interpretó el cuadro como una neumonía y se indicó ceftriaxona. Una broncofibroscopia (BFC) reveló un gran tapón mucoso, duro, que ocluía la entrada de los dos lóbulos. Se logró permeabilizar parcialmente el bronquio originándose un sangrado importante. $\mathrm{Al}$ hacerlo, drenó abundante secreción purulenta. Posterior a ello hubo reexpansión del pulmón izquierdo y la enferma fue dada de alta 15 días después. No se efectuó una tomografía computada en esa hospitalización.

Un mes después del alta, la enferma consultó en un servicio de urgencia por disnea progresiva, tos y expectoración mucosa. Nuevamente se constató abolición del murmullo pulmonar y matidez del hemitórax izquierdo con velamiento del mismo en la radiografía de tórax. Persistía la anemia y existía leucocitosis de $17.700 \mathrm{~mm}^{3}$. Se indicó cefuroxima, kinesioterapia respiratoria intensiva y nebulizaciones con fenoterol y bromuro de ipatropio. Con ello se logró la reexpansión del pulmón izquierdo. Días después se reprodujo la atelectasia, con velamiento completo del pulmón izquierdo y desviación de la tráquea ipsilateral (Figura 1). Una BFC mostró que la tráquea y el árbol bronquial derecho estaban normales y en el bronquio izquierdo, a $2 \mathrm{~cm}$ de la carina principal, existía una masa blanquecina, cerebroidea que obstruía completamente el lumen impidiendo el paso del broncofibroscopio. La citología reveló células malignas y la biopsia, una mucosa bronquial infiltrada por un adenocarcinoma tubular moderadamente diferenciado, con aspecto de un carcinoma colónico. La inmunohistoquímica fue positiva para citoqueratina 20 y negativa para citoqueratina 7, apoyando al origen colónico del tumor. Una tomografía computada de tórax evidenció un tumor que ocupaba completamente el bronquio fuente izquierdo (Figura 2), adenopatías mediastínicas bilaterales y un quiste hidatídico calcificado en el lóbulo izquierdo del hígado.

Se le propuso radioterapia, lo que fue rechazado por la paciente. Egresó con indicaciones de kinesioterapia domiciliaria y uso de oxígeno según necesidad. La enferma, a 11 meses de su alta, sobrevive sin haber requerido nuevas hospitalizaciones.

\section{DisCUSIÓN}

El hallazgo de metástasis EB de un cáncer extrapulmonar es poco frecuente ${ }^{1,4}$. Unos autores en una búsqueda en MedLine encontraron 204 casos publicados entre 1966 y $2002^{3}$. En un estudio retrospectivo realizado en hospitales mexicanos en 2.836 BFC efectuadas en pacientes con antecedentes de tumores malignos extrapulmonares, encontraron 58 sujetos con metástasis $E^{5}$. Otro estudio, prospectivo, realizado en el Reino Unido durante 15 años, en 3.353 BFC encontró 1.391 con anormalidades EB. De ellas, en 1.059 la biopsia reveló un tumor maligno y sólo $16(1,5 \%)$ correspondieron a una

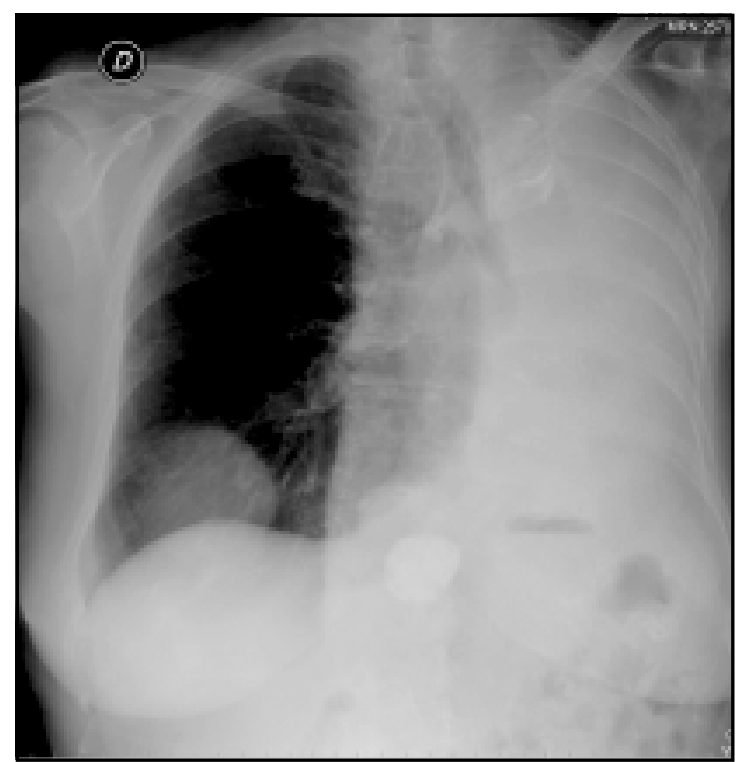

Figura 1. 


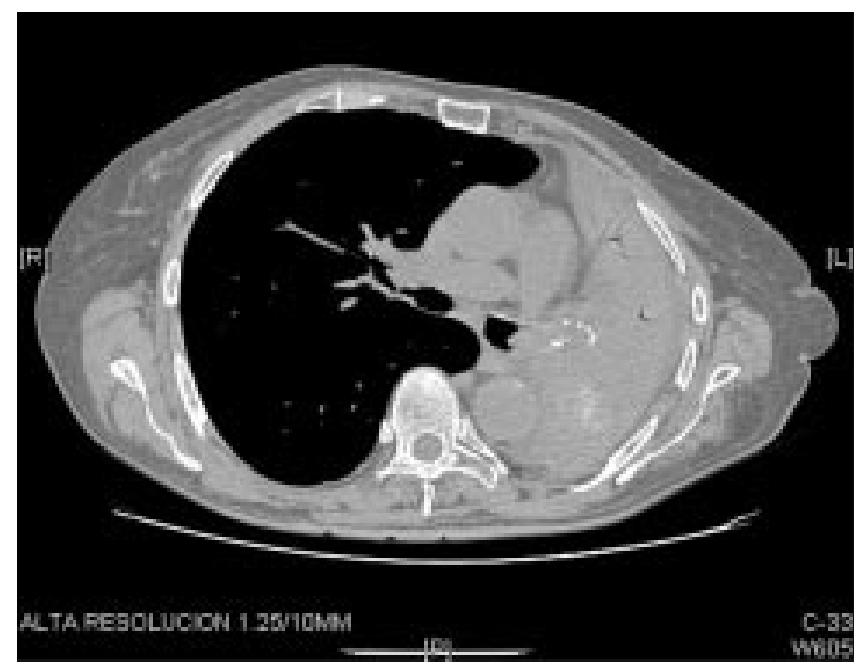

Figura 2.

metástasis EB. En estas series fue más frecuente el origen mamario y renal que el colorectal.

En Chile encontramos sólo una publicación de 2 pacientes con metástasis endobronquiales, una de origen mamario y otra colónica, los que fueron tratados con terapia fotolumínica ${ }^{6}$.

El modo de diseminación hacia un bronquio no está completamente establecido. Se han propuesto cuatro alternativas: Tipo I: metástasis directa al bronquio; Tipo II: invasión bronquial desde una lesión parenquimatosa; Tipo III: invasión desde adenopatías metastásicas hiliares o mediastínicas y Tipo IV: lesiones periféricas extendidas a través de los bronquios principales. En el cáncer colorrectal se han propuesto como las formas de diseminación más probables las tipo I, II y IV ${ }^{7}$.

Los síntomas de presentación más frecuentes son la disnea (18-80\%), tos (37-66\%) y hemoptisis (26-33\%). Excepcionalmente pueden presentarse por la expectoración de un tejido sólido, cuyo análisis histológico revela un adenocarcinoma colónico ${ }^{8}$. También puede ser un hallazgo en sujetos asintomáticos con radiografías de tórax alteradas. Radiológicamente las metástasis EB pueden presentarse como una masa tumoral (2641\%), nódulos múltiples (17-40\%) o atelectasias $(17-58 \%)^{2,3,9,10}$, como sucedió en esta paciente. El escáner tiene una mayor sensibilidad que la radiografía de tórax para la detección de metástasis pulmonares y $\mathrm{EB}^{11}$.
La BFC es la forma más frecuente de hacer el diagnóstico y tiene una sensibilidad cercana a $100 \%$ en las lesiones centrales. Son características el aspecto de trombo, la obstrucción de los orificios bronquiales cercanos y su fuerte tendencia al sangrado ${ }^{12,13}$. En las lesiones más distales el diagnóstico se hace por la citología obtenida por el cepillado o el lavado bronquial. Otra forma de hacer el diagnóstico de metástasis EB es el estudio citológico de la expectoración ${ }^{14}$.

La mayoría de los casos de metástasis EB ocurre en pacientes con un carcinoma colorrectal conocido ${ }^{15}$, pero se han comunicado también en sujetos en que se desconocía su existencia ${ }^{16}$. El intervalo transcurrido entre el diagnóstico del cáncer primario y el de la metástasis EB ha variado entre unos pocos meses hasta 17 años ${ }^{17}$. En el cáncer de colon, éste es en promedio de 26 meses y en el de recto 32 meses $^{18}$.

La sobrevida una vez diagnosticada la metástasis EB puede llegar a 21 meses, cuando se efectúa un tratamiento quirúrgico local ${ }^{19}$, pudiendo extenderse en promedio hasta 3,5 años con una resección quirúrgica amplia ${ }^{20}$. Otras terapias empleadas por vía endoscópica han sido la braquiterapia, láser, crioterapia, implantes de stents y el tratamiento fotodinámico ${ }^{6}$. En $17 \%$ de los pacientes, como lo fue este caso, dado el rechazo de la enferma, no es factible realizar ningún procedimiento terapéutico ${ }^{3}$. 
En suma, comunicamos el caso de una anciana con una atelectasia pulmonar izquierda recurrente secundaria a una metástasis endobronquial de un cáncer de colon operado 3 años antes, que fue inicialmente interpretada como producida por un tapón mucoso. El antecedente de un tumor malig-

\section{REFERENCIAS}

1. Braman S, Whitcomb M. Endobronchial metastasis. Arch Intern Med 1975; 135: 543-7.

2. Flores M, Corzo F, Bazán G, Lemus Z, Zubiate C, García $K$. Incidencia de enfermedad endobronquial metastásica en un hospital oncológico. Gamo 2005; 4: 88-92.

3. Jens B. Endobronchial metastases from extrapulmonary solid tumors. Acta oncológica 2004; 43: 73-9.

4. Mileron B, Jean T, Fouret P, Liote H, Akoun G. Endobronchial metastases of cancer. A propos of 29 cases. Rev pneumol clin 1986; 42: 231-4.

5. Tsukamoto T, Nagasana M, Yamada $K$, Satoh $T$. Endoscopic endobronchial metastasis. Nihon kyobu shikkan gakkai zasshi. 1992; 30: 609-13.

6. Caviedes I, Badinez L, Cordova A, Goset K, Zeiada S. Terapia fotodinámica: aplicación en dos pacientes con metástasis endobronquiales de adenocarcinoma extrapulmonar. Rev Chil Enf Respir 2003; 19: 38-42.

7. Kiryu T, Hoshi H, Matsui E, Iwata H, Kokubo M, SHIMOKAWA K ET AL. Endotracheal/Endobronchial metastases. Chest 2001; 119: 768-75.

8. Zias E, Owen R, Borezuk A, Reichel J, Frater R. An unusual presentation of metastatic colon cancer to the lung. Chest 1998; 113: 244-6.

9. Dursun A, Demirag F, Bayiz H, Sertkaya D. Endobronchial metastases: a clinicopathological analysis. Respirology 2005; 10: 510-4.

10. Foon B, Fung-Gi I, Fin Mo G, Myung F, Mi-Young K. Atypical pulmonary metastases: spectrum of radiologic findings. Radiographics 2001; 21: 403-17. no extrapulmonar debe hacer pensar en una metástasis EB frente a una atelectasia de causa no explicada. El envío del tapón mucoso obtenido en la BFC para el análisis histopatológico permite el diagnóstico de esta patología cuando clínicamente no se ha sospechado.

11. Herold C, Bankier A, Fieischmann D. Lung metastases. European Radiology 1996; 6: 596-606.

12. Heitmiler R, Marasco W, Hruban R, Marsh B. Endobronchial metastasis. J Thorac Cardiovasc Surg 1993; 106: 537-42.

13. Oshikawa K, Ohno S, Ishi $Y$, Kitamura S. Evaluation of bronchoscopic findings in patients with metastatic pulmonary tumor. Intern Med 1998; 37: 34953.

14. Radford D, Petrem N, Herrera L, Gamarra M. Sputum cytology for the detection of pulmonary metastases fron colorectal carcinoma. Dis colon rectum 1987; 30: 678-82.

15. Mourux J, Bereder J, Benchimol D, Bernard J, Bourgeon A, Richelme H. Pulmonary metastases of colorectal origin. Chirurgie 1990; 116: 53-8.

16. IshiKawa A, МотоHashi $S$, ShibUya $K$, Baba $M$, TOYOSAKI T, OHWADA H ET AL. Small solitary pulmonary metastasis is detected before primary sigmoid colon cancer: report of a case. Surg Today 2003; 33: 709-11.

17. SHEPHERD M. Endobronchial metastatic disease. Thorax 1982; 37: 362-5.

18. Sebaha A, Eyup S, Gulperi C, Gulper S, Oguz K, Oya I. Endobronchial metastases from extrathoracic malignancies. Clin Exp Metastasis 2005; 22: 58791.

19. Carlin W, Harrell H, Olson K, Moser M. Endobronchial metastases due to colorectal carcinoma. Chest 1989; 96: 1110-4.

20. Mansel K, Zinsmeister R, Pairolero $\mathrm{C}$, Jett $R$. Pulmonary resection of metastatic colorectal adenocarcinoma. A ten year experience. Chest 1986; 89: 109-12. 